\title{
ELEMENTI DI ICNOGRAFIA MEDITERRANEA (PROTONURAGHI A CAMERA NAVIFORME)
}

\author{
POR \\ LUCIA MANCA DEMURTAS \\ SEBASTIANO DEMURTAS
}

RESUMEN Definimos como protonuraghi con cámara «naviforme» aquellos nuraghi caracterizados por un corredor o espacio principal que, por ampliación de las paredes laterales, se transforma en cámara. Esta presenta la parte posterior en forma de ábside semicircular y la cubierta en ojiva.

El tipo, ya examinado por nosotros en el ámbito de un análisis general, aunque preliminar, sobre los protonuraghi, parece, por el momento, preludiar, en una propuesta de evolución arquitectónica, al nuraghe en tholos.

Nuestro análisis no intenta fijar los puntos cronológicos en el interior del paso evolutivo aunque parece presumible que la creación y la difusión de este tipo de monumento pueda encuadrarse entre el Bronce antiguo y el Bronce medio teniendo en cuenta también los datos materiales adquiridos hasta ahora.

Aunque sin infravalorar un posible aporte externo en la definición del tholos, parece igualmente evidente que la aceptación de tal modelo arquitectónico no pudo ser tan contrastante con la experiencia ya adquirida.

RIASSUNTO Definiamo protonuraghi con camera "naviforme" quei nuraghi il cui elemento caratterizzante è il corridoio o spazio principale che per l'ampliamento delle pareti laterali si muta in camera. Essa presenta la parte posteriore in forma di abside semicircolare e la copertura ad ogiva.

Il tipo, da noi già preso in esame nell'ambito di un'analisi generale, seppure preliminare, sui protonuraghi, sembrerebbe, al momento, preludere, in una proposta di evoluzione architettonica, al nuraghe a tholos.

La nostra analisi esula dall'intento di fissare dei punti cronologici all'interno del passaggio evolutivo anche se appare presumibile che la creazione e la diffusione di questo tipo di monumento possa inquadrarsi tra il Bronzo antico e il Bronzo medio tenendo conto anche dei dati materiali fin'ora acquisiti.

Anche non sottovalutando un possibile apporto esterno nel definirsi della tholos, appare altrettanto evidente che l'accettazione di tale modulo architettonico non può essere così contrastante con le esperienze già acquisite.

Palabras clave Protonuraghi con cámara «naviforme». Tholos. Bronce Antiguo. Bronce Medio. Cerdeña.

Parola chlave Protonuraghi con camera knaviforme». Tholos. Bronzo antico. Bronzo medio. Sardegna. 


\section{INTRODUZIONE (*)}

Si cercherà di condurre un' analisi architettonica dettagliata di un tipo di monumento appartenente alla tipologia dei protonuraghi da noi definiti con camera «naviforme" (1).

La peculiarità di questi monumenti è costituita dall'ambiente principale, che occupa uno spazio rilevante, determinato dall'ampliarsi delle pareti laterali del corridoio e dal progressivo elevarsi della copertura che si innalza verso il centro chiudendo ad ogiva tronca o a cuneo, con il limite della parete posteriore ad abside semicircolare. Allo stato attuale della ricerca se ne possono individuare sette: Sa Jacca-Busachi (Tav. I, 1), Orgono-Ghilarza (Tav. I, 2), S. Antine e Campu Sedilo (Tav. I, 3), Crastu A-Soddi (Tav. I, 4), Fodde-Bolotana (Tav. I, 5), Conculas-Macomer (Tav. 1, 6), CorcoveOrotelli (Tav. 1, 7). Sono per ora localizzati in una zona molto circoscritta, nella Sardegna centrale, che tocca i lembi del Marghine, l'altopiano di Abbasanta, la piana di Macomer e il Barigadu.

Di fatto, generici riferimenti a questi monumenti si hanno in diversi studiosi. Nel $1886 \mathrm{fu}$ il Centurione a dare notizia del nuraghe Crastu (Ghilarza). Presentandone la pianta e la sezione fornisce dei dati strutturali e formali (2). Nel 1904 il Taramelli descrive il nuraghe Congiau Cotta o Sa Jacca (Busachi) rilevando la particolarità della sua forma che "è allargata in forma di prora di nave" (3). Si possono, poi, ricordare brevi citazioni per qualcuno di essi come Orgono (4) e di recente qualche fonte indicativa per Conculas e Crastu, mentre per il nuraghe Orgono abbiamo una descrizione dettagliata (5). Tali strutture vengono però definite nuraghi ma, invece, date di recente come protonuraghi (6) per i quali si è proposto un primo tentativo di classificazione basantesi sulla presenza del corridoio e sulla sua riduzione funzionale dato il sopravanzare della camera come spazio principale (7).

\section{SCHEDE}

\section{Sa Jacca-Busachi-OR (Tav. I, Tav. III, 2)}

E'situato a m. 316 d'altitudine su un affioramento granitico. (F. 206, quadr. II, S-E, Busachi).

II nuraghe presenta un ingresso, una camera naviforme (C), tre ambienti (A, B, D) disposti in posizione bilaterale lungo il corridoio e vano scala. Ha un contorno planimetrico irregolare con l'addossamento alla roccia nel lato volto a Ovest-Sud-Ovest. La parete in cui si apre l'ingresso è tronca sul lato destro, con la chiusura a spigolo ad Est, mentre nella sua parte opposta (lato sinistro)

(*) Ringraziamo il Soprintendente Archeologo Dottor Vicenzo Santoni per la liberalità con cui ci ha concesso di utilizzare la planimetria del nuraghe Fodde e condurre il relativo studio.

(1) Definiamo protonuraghi con camera «naviforme" quei nuraghi che per il tipo di pianta interna si possono confrontare con le nau, monumenti megalitici delle Baleari. Nel 1818 fu Don Yuan Ramis y Ramis che per primo diede il nome di nau o navetas a delle costruzioni di Minorca, in forma di nave. In particolare si riferiva alla nau di Els Tudons: «La parte central de la figura hay una doble linea longitudinal que pretende representar la cresta de la quilla de la nave. (Ramis y Ramis 1818 apud Veny, 1982: 181-183). Della distinzione funzionale e strutturale utilizzata dagli studiosi spagnoli che indicano col termine «nau» la naveta-tomba minorchina e con «naviforme» o *navetiformew la naveta-abitazione (Veny, 1982: 207) non deriva per l'analisi dei nostri monumenti una differenziazione di pianta. Pertanto la nostra analisi prescinderà dall'utilizzo anche se non esistono elementi per definirlo altro che non sia abitativo. In tale occasione si intende precisare anche la suddetta tipologia anteriormente definita "navetiforme" (Manca-Demurtas, 1984 a: 635 e 1984 b: 174).

(2) Centurione, 1886: 188-190, figs. XXIII, XXIV, XXV.

(3) Taramelli, 1904: 218, fig. 9.

(4) Centurione, 1886: 191; E.E.M., 1922: 116.

(5) Conculas: Santoni, 1980: 143, fig. 133, 38; Crastu: Santoni, 1980: 160, fig. 152; Lilliu, 1982: 15, fig. 4, 2; Orgono: Contu, 1981: 23, figs. 10-11; Manca, 1985: 16-17.

(6) Manca-Demurtas, 1984 a: 631 e 1984 b: 167.

(7) Manca-Demurtas 1984 a: 635 e 1984 b: 173, 174. 


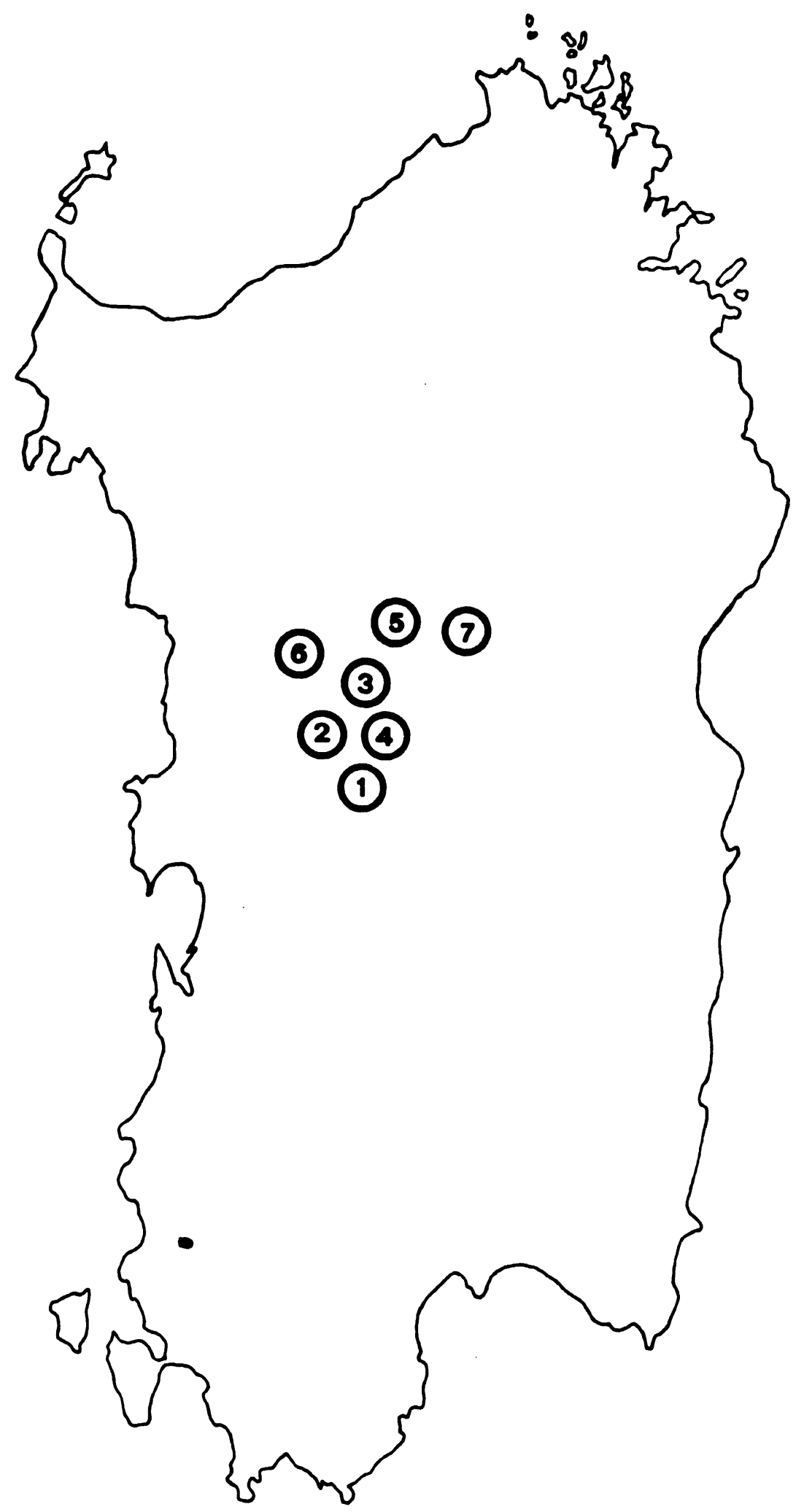

TAv. 1.-Distribuzione dei protonurahi a camera naviforme. 1) Sa Jacca Busachi (Oristano). 2) Orgono Ghilarza (Oristano). 3) S. Antine e Campu Sedilo (Oristano). 4) Crastu A Soddi (Oristano). 5) Fodde Bolotana (Nuoro). 6) Conculas Macomer (Nuoro). 7) Corcove Orotelli (Nuoro). (S. Demurtas). 
risalta la linea ondulata. Anche la parete contrapposta, in prossimità di quella pertinente all'apertura $\mathrm{b}$, ha un profilo arrotondato con una parte terminale (a Nord-Est) più rigida. Il corridoio si apre a Sud-Est e si contiene nella massa muraria, lungo m. 3,50 e largo m. 1,20. La stessa larghezza si misura all'ingresso (larghezza alla base $\mathrm{m} .1,20$, in alto $1 \times$ altezza residua 1,10 ), munito di un architrave rettangolare, ben curato (lunghezza m. $2,15 \times$ larghezza $0,70 \times$ spessore 0,50 ) e sormontato da un finestrino di scarico, anch'esso rettangolare (altezza m. 0,55 $\times$ larghezza 0,30). L'altezza del corridoio è di $\mathrm{m}$. 1,80 dopo l'architrave dell'ingresso. Procedendo verso l'interno esso si innalza con una copertura a piattabanda regolare ascendente fino a raggiungere $\mathrm{m}$. 2,80 all'incontro con la scala e l'inizio della camera naviforme. A m. 1,40 dall'ingresso si apre la cella A, di forma semiellittica, larga nella parte iniziale $\mathrm{m} .0,70$ e profonda $\mathrm{m}$. 2,70. Il corridoio nel suo primo tratto di $\mathrm{m} .3,50$ ha un percorso rettilineo, poi procede con uno slargo delle pareti costituendo un passaggio di maggiore ampiezza e raggiungendo in larghezza m. 2,10 nel punto in cui inizia l'ambiente B e m. 2,40 nel limite contiguo allo spazio $D$. Questo spazio maggiore $(C)$ o camera naviforme ha una lunghezza di m. 4,20/4,30 ed un altezza di m. 4,40. Sulla stessa linea di A, a m. 2, si apre l'imbocco della scala (larghezza m. $1 \times 3$ altezza) con un andamento curvilineo e a m. 1,40 da questo, il vano B. Questo, largo al suo imbocco m. 1,75, si articola in due parti differenziate: uno spazio più ampio emiellissoidale il primo (B: profondità $\mathrm{m} .2 \times 3$ altezza) ed uno stretto passaggio pressoché quadrangolare che prende luce dall'esterno, il secondo (b: larghezza alla base $\mathrm{m} .1,20 \times 0,50$ in alto $\times 1,10$ altezza).

Sul fondo della camera $C$, costituito dalla roccia naturale livellato a parete, sulla sinistra, si apre uno spazio sub-circolare (D: larghezza alla base m. 1,25 in alto 0,50 $\times$ altezza $2,20 \times$ profondità 1,30), architravato (architrave triangolare: lunghezza $\mathrm{m} .1 \times 0,50$ altezza). Gli interni di tutti gli ambienti presentano una tecnica muraria a filari irregolari. I blocchi impiegati sono di grandi e medie dimensioni variamente poliedrici. In particolare nella camera $C$ ai blocchi si interpongono lastroni appiattiti. Abbondante è la rinzeppatura con piccole pietre. Le pareti aggettano fortemente verso l'alto chiudendo ad ogiva tronca. Anche nello spazio D e B la copertura è ad ogiva tronca, mentre in b è a lastroni piattabandati. Si segnala il dato strutturale della corrispondenza dell'uso della trachite per l'imposta e la composizione della copertura, quando si tratti del tipo ad ogiva tronca; del granito, invece, per la copertura a piattabanda e per la struttura basale. Esternamente, nella linea dell'ingresso, oltre il finestrino di scarico, si contano cinque filari residui. Il monumento nel suo stato di conservazione è in discrete condizioni.

BIBLIOGRAFIA: (Taramelli, 1904: 218, fig. 9; Santoni, 1976: 38; Idem, 1980: 146, fig. 137; Lilliu, 1982: 32, fig. 24; Manca-Demurtas, 1984 a: 633, fig. 1, 24 e 1984 b: 169, fig. 1, 24. (OR): 180).

\section{Corcove-Orotelli-NU (Tav. I, Tav. II, 2)}

Situato a m. 391 d'altitudine, il monumento poggia su un affioramento roccioso granitico (F. 207, quadr. IV, N-E, Orotelli).

Ha una forma quadrangolare irregolare con un'espansione di m. 12 nell'asse maggiore a NordSud e di m. 8 nell'asse minore a Est-Ovest. La giacitura di base e l'addosamento alla roccia obbligano il monumento ad assumere una figura irregolarmente quadrangolare con il lato da Nord ad Ovest, parzialmente inglobato nella roccia, a profilo curvilineo, ed il lato da Nord ad Est costituito da segmenti curvilinei e retti che si concludono nel lato a Sud in una leggera concavità con apice arrotondato. E'costituito da un ingresso, una camera naviforme, due celle a sviluppo unilaterale e cella terminale lungo l'asse del corridoio. L'ingresso si apre a Sud ed è decentrato verso Ovest. Il corridoio largo m. 0,80 si allarga gradatamente fino a m. 1,50 nel punto in cui si apre sulla destra la cella A, e fino a m. 2,70 nell'incontro con la cella B. Nel suo primo percorso di m. 4 ha un andamento piuttosto lineare e continua con uno svasamento progressivo "ad imbuto" per assumere poi una forma "naviforme" e costituire una camera o vano principale che si allunga per $\mathrm{m}$. 8. Attualmente il primo braccio d'andito è molto rovinato per cui non è possibile dare dettagli nè dell'altezza nè dei contorni parietali. La disposizione delle celle A (larghezza m. 0,65/0,90 al centro $X$ 

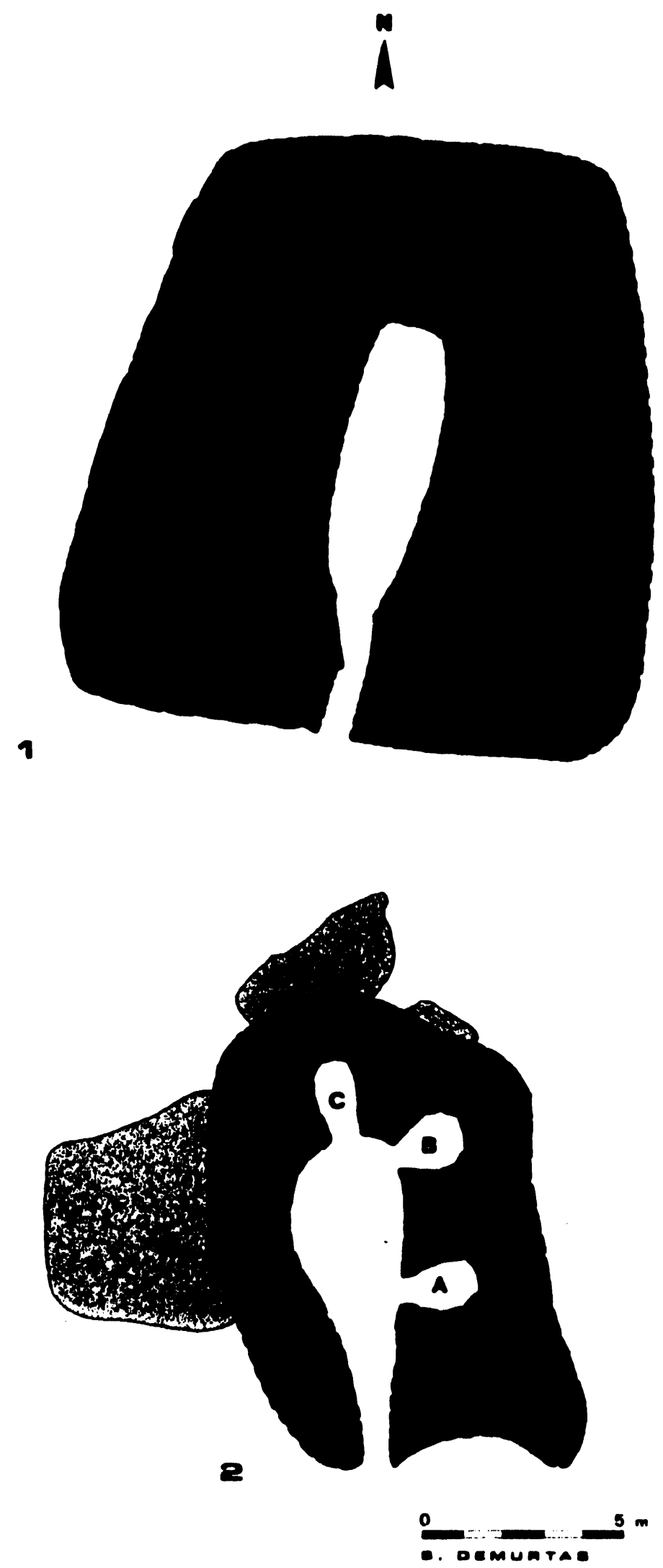

Tav. 2.-Naviformi: 1) Fodde Bolotana (NU) Pianta. 2) Corcove Orotelli (NU) Pianta. (Ril e Dis. S. Demurtas).

T. P., $1991, \mathrm{n}^{2} 48$ 
2 profondità), B (larghezza $\mathrm{m}$. 0,65/1,30 al centro $\times 1,60$ profondità $\times 1,50$ altezza residua), $\mathrm{C}$ (larghezza $\mathrm{m} .0,65 / 1$ al centro $\times 2$ profondità) avviene nella parte centrale della camera. A e B hanno uno sviluppo unilaterale distanziate tra loro m. 2,30; alla breve distanza da B di m. 1,20 nella parte terminale del corridoio, nella stessa direzione dell'asse longitudinale dell'ingresso, si apre la cella $\mathrm{C}$.' Tutte e tre le celle sono molto rovinate; si nota il contorno che è semiellittico e residuano solo in parte i filari delle pareti. Anche la camera principale è molto rovinata. Si presuppone avesse una copertura ad ogiva tronca. Il monumento si trova in pessime condizioni sia esternamente che internamente. Le pareti murarie che residuano mettono in evidenza un'opera poliedrica a filari non régolari. I blocchi usati sono di medie dimensioni con abbondante rinzeppatura. Nel lato volto ad Ovest, oltre la parete rocciosa su cui si imposta l'opera manufatta, residuano quattro filari per un'altezza di m. 2.

BIBLIOGRAFIA: (Santoni, 1965-1966: 113; Idem, 1976: 38 Idem, 1980: 143, fig. 133, 28: 158, fig. 149; Lilliu, 1982: 15, fig. 3; Manca-Demurtas, 1984. a: 638 e 1984 b: 174).

\section{Fodde-Bolotana-NU (Tav. I, Tav. II, 1)}

E'situato a circa m. 300 d'altitudine su un'appendice collinare, nei pressi del Rio Cannas (F. 207, quadr. IV, N-O, Bolotana).

Il nuraghe presenta un ingresso e una camera naviforme. Ha una forma planimetrica trapezoidale irregolare. E'rilevabile solo il lato occidentale, mentre meno chiara appare la linea di contorno esterno sui rimanenti lati con parziale suffetazione nella parte orientale. Ha una lunghezza di $\mathrm{m}$. 14,80 lungo l'asse longitudinale, di m. 12,40 circa lungo l'asse trasverso. La parete in cui si apre l'ingresso è rettilinea, lievemente arrotondato nello spigolo ad Ovest-Sud-Ovest. L'ingresso (largo m. 0,90) si apre a Sud. E'munito di architrave (lunghezza m. 1,60 $\times 0,50$ spessore; larghezza non definibile) ed immette in uno stretto corridoio rettangolare (lungo m. 3,30), accesso allo spazio centrale a vano allungato, in schema naviforme. Al termine del corridoio, in parte crollato, si individuano alcune lastre di copertura. II vano è lungo m. 7 e largo al centro m. 2,40; attualmente ricolmo di materiale di riempimento è difficile calcolare le dimensioni anche se è presumibile un'altezza residua di $\mathrm{m} .4,60 / 4,80$. Residua la parete laterale sinistra per m. 1,10 e quella di fondo per $\mathrm{m}$. 1,60. Questa chiude ad abside e si raccorda con le pareti laterali aggettanti verso l'alto e chiudenti presumibilmente ad ogiva tronca. La tecnica muraria appare trascurata per l'interno, meno per l'esterno. All'esterno si mette in evidenza un sistema filarico di blocchi che sono di notevoli dimensioni soprattutto nell'imposta di base. Essi procedono verso l'alto, variamente poliedrici e di medie dimensioni, a linee discontinue, adagiandosi talvolta su piani obliqui. Nel lato volto ad Ovest, i massi del primo filare sono disposti a coltello con poche zeppe di rincalzo. All'interno, nella camera, si osserva che i blocchi sono disposti per ordine orizzontale alternati ad altri obliqui, sempre di punta, ma prevale la giacitura obliqua. Frequenti sono le zeppe di rincalzo. E'difficile dire se dalla camera si diparta la scala d'accesso alla parte superiore. L'altezza massima esterna calcolabile è nel lato volto ad Ovest ed è di $\mathrm{m}$. 5 , con un'inclinazione accentuata della parete di $\mathrm{m}$. 1,25 . Il materiale usato per la costruzione è il basalto.

BIBLIOGRAFIA: (Santoni, 1976: 38; Idem, 1980: 143, fig. 133, 14; Manca-Demurtas, 1984 a: 638 e 1984 b: 174).

\section{Conculas-Macomer-NU (Tav. I, Tav. III, 1)}

E'situato a m. 414 d'altitudine, in una vallata, su un rialzo roccioso. A m. 50, a Sud, scorre il rio Tossilo (F. 206, quadr. I, S-O, Borore).

Il protonuraghe è provvisto di un ingresso, camera naviforme $(\mathrm{A})$, tre celle $(\mathrm{B}, \mathrm{C}, \mathrm{D})$ delle quali

\section{T. P., 1991, $\mathrm{n}^{9} 48$}


una (B) con corridoio a svolto angolare, vano scala. La pianta, all'esterno, mette in evidenza una forma ovale con un'espansione di m. 13 nell'asse maggiore a Nord-Sud, di m. 10,50 nell'asse minore ad Est-Ovest. L'ingresso si apre ad Est. Di luce trapezoidale, é sormontato da un lastrone di forma rettangolare (larghezza alla base $\mathrm{m}$. 1,20/1 alla sommità $\times 1,70$ altezza; architrave: $\mathrm{m} .1,60 \times 0,50 \times$ 0,90 in lunghezza, altezza e profondità). Il corridoio, che si contiene nella massa muraria, é decentrato verso Nord e taglia l'asse trasverso della costruzione per una lunghezza di m. 8,50. La sua larghezza aumenta gradatamente da m. 1,20 a m. 1,80 nella parte centrale per diminuire progressivamente nel limite fondale dove chiude con un profilo arrotondato. Il corridoio che funge da camera o vano principale (A), è fiancheggiato lateralmente dalle celle B e C. Queste sono disposte non simmetricamente ai suoi due lati determinandosi cosí uno schema a transetto semplice, articolato nella cella $C$ a sviluppo tangenziale e nella cella $B$ parallela all'asse trasverso ed unita ad A da un breve passaggio d'accesso b. Infatti, il vano A, a m. 3,50 dal suo imbocco e con una larghezza di m. 1,80, da adito, a sinistra, a questo braccio d'andito lungo m. 3 nel suo lato maggiore che, per m. 1,70, si allarga gradamente da m. 1,20/1,30 mantenendo di seguito tale larghezza per svoltare a sinistra ad angolo ed incontrare la cella B, di forma semiellittica (lunghezza m. 2,20 $\times 1,40$ larghezza) mentre nel suo percorso lineare chiude nella parete di fondo con un'altra cella $\mathrm{D}$ (lunghezza $\mathrm{m}$. 2,00 $\times 1,20$ larghezza). L'opera muraria interna appare curata nella scelta delle pietre. Queste, pur al naturale, prive di lavorazione e variamente poliedriche, sono saldamente legate tra loro con un incastro a interblocco dove è marcata la rinzeppatura. L'inclinazione delle pareti è accentuata e la copertura doveva essere piattabandata. Nel braccio d'andito b, la cui parete sinistra (rispetto a chi entra) misura m. 2,70, i blocchi impiegati sono di maggiori dimensioni e per lo più rozzi che non indicano un preciso ordine filarico. Per la situazione di crollo non si può determinare l'altezza ne è visibile la copertura che si presume fosse a piattabanda per la presenza di enormi lastre giacenti. In tale andito, sulla destra, quasi di fronte a B, si apre il vano scala con sviluppo a gomito (larghezza $\mathrm{m}$. 1,10). A m. 4,50 dall'ingresso, sulla destra, si ha l'apertura della cella $C$ (larghezza alla base $\mathrm{m}$. 1,40 $\times$ 2,10 profondità). Essa è molto rovinata. E'leggibile, comunque, il contorno che è semiellittico. Nel vano o camera naviforme è assai marcato l'aggetto progressivo delle pareti ottenuto con la giacitura obliqua dei lastroni che si innalzano per cinque filari. Essi sono rozzi tenuti saldi da piccole zeppe di rincalzo. Il profilo della chiusura è presumibilmente ad ogiva tronca. Lo stato di conservazione è relativamente discreto per quanto riguarda l'esterno. Per l'interno e la parte alta si ha crollo e riempimento. Esternamente è leggibile in operà l'intera struttura edilizia in cui l'altezza residua è di m. 3,50.

Si nota una tecnica muraria molto rozza: $\mathrm{i}$ massi, poggianti in qualche punto (lato Nord) direttamente sulla roccia, sono molto irregolari formando filari discontinui. Vari sono gli spazi vuoti con poche zeppe di rinforzo. Nel lato volto a Nord si contano sei filari, negli altri in media quattro/cinque. Il materiale usato è il basalto.

BIBLIOGRAFIA: (Santoni, 1980: 143, fig. 133, 38; Manca-Demurtas, 1984 a: 638 e 1984 b: 174: 179, fig. 8, c).

\section{S. Antine e Campu-Sedilo-OR (Tav. I)}

E'situato a m. 318 d'altitudine su una dorsale (F. 206, quadr. I, S-E, Sedilo).

Il nuraghe presenta un ingresso, una camera naviforme, tre celle $(A, B, C)$ di cui due parzialmente simmetriche (B, C) e la prima (A) disposta sullo stesso lato della seconda (B). Ha una forma planimetrica ellittica tronca con un diametro approssimativo in senso longitudinale di $\mathrm{m}$. 14,50 e di m. 14 in senso trasverso. La fronte in cui si apre l'ingresso è rettilinea, lunga m. 8,80. Dell'ingresso si possono determinare solo le misure dell'architrave, triangolare $(\mathrm{m} .1,45 \times 0,65 \times 0,70$ in lunghezza, altezza e profondità). Un breve corridoio (lunghezza presumibile m. 3,10), attualmente ostruito ma leggibile nella parte alta, porta alla camera, alla quale si può accedere dall'alto attraverso un varco moderno poichè mancano alcuni lastroni di imposta della copertura. La camera è lunga $\mathrm{m}$. 7,70 e 

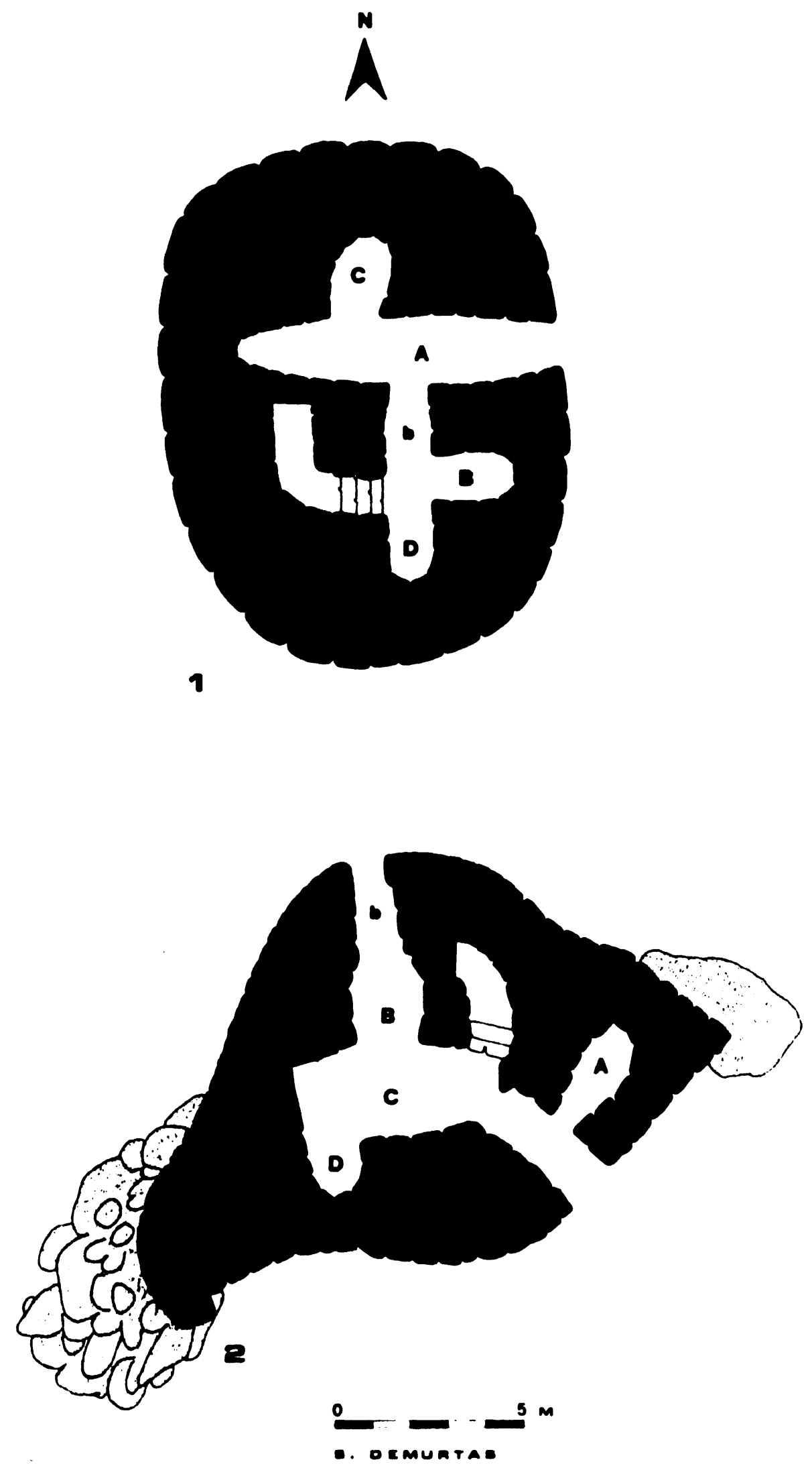

TAv. 3.-Naviformi: 1) Conculas Macomer (NU) Pianta. (Ril E Dis. S. Demurtas) 2) Sa Jacca Busachi (OR) Pianta. (Da Santoni 1980, p. 146, Fig. 137). (Dis. S. Demurtas).

T. P., 1991, $\mathrm{n}^{9} 48$

(c) Consejo Superior de Investigaciones Científicas

http://tp.revistas.csic.es

Licencia Creative Commons 3.0 España (by-nc) 
larga al centro m. 2,85 e chiude la parete di fondo in forma di abside semicircolare. Forte è l'aggetto delle pareti dove si contano dieci filari costituiti da pietre di medie dimensioni che diminuiscono di grandezza verso l'alto. Si raggiunge al centro la massima altezza di $\mathrm{m}$. 5 con una chiusura a cuneo. Distante m. 1,20 dalla linea del corridoio sulla destra, si apre la cella A, di forma semiellittica (larghezza al centro m. 1,45 $\times$ profondità $2,70 \times$ altezza 2 ). Sullo stesso lato di A a m. 4,60 da esso, si trova la cella B, anch'essa di forma semiellittica (larghezza alla base m. 0,90 al centro 1,60 $\times$ profondità $2,70 \times$ altezza 1,70), munita di architrave triangolare $(\mathrm{m} .0,80 \times 0,35 \times 0,70$ in lunghezza, altezza e profondità). Di fronte a B,a circa m. 2,10 in linea, si apre la cella $C$, di forma ellittica, con una profondità di m. 2,40. Le celle chiudono con una copertura a lastroni. La tecnica muraria sia interna che esterna è molto rozza con l'uso di grandi blocchi che vanno diminuendo di dimensioni verso l'alto. Le forme variamente poliedriche sono proporzionate per la sovrapposizione ed il legamento dei blocchi laterali. La parete volta a Nord residua per tre/quattro filari per m. 2,50 con una inclinazione di m. 0,50. Il monumento si presenta in discrete condizioni. Il materiale usato per la costruzione è il basalto.

\section{BIBLIOGRAFIA: Inedito}

\section{Orgono-Ghilarza-OR (Tav. I, Tav. IV)}

E'situato a m. 248 d'altitudine, in un altopiano. A m. 50 circa ad Est, nel vallone sottostante, scorre il fiume Messe Cappai. (F. 206, quadr. II, N-E, Ghilarza).

La costruzione è di tipo misto: al primitivo corpo che è un protonuraghe si aggiunge nella parte alta un corpo a tholos. Un rifascio cinge la cinta muraria con lo sbocco di una cella a svolta angolare. Il protonuraghe presenta un ingresso, una camera naviforme, due celle bilaterali (A e B) e vano scala (C). Il corpo a tholos è costituito da una camera circolare (D). L'intera struttura ha un profilo ovoidale includendo il rifascio per un diametro maggiore di $\mathrm{m} .17,80$, minore di $\mathrm{m}$. 13,60. La planimetria del corpo primitivo mostra un corridoio, che si contiene nella massa muraria con funzione di camera di forma naviforme (lunghezza m. 8,30 $\times$ larghezza 1,20/2,60). Essa è fiancheggiata lateralmente da due celle (A e B). L'ingresso, rivolto a Sud-Sud-Est, é di luce trapezoidale (larghezza alla base $\mathrm{m} .1 .20 / 0,90$ in alto $\times 1,50$ altezza) e sormontato da un robusto lastrone di architrave, di forma rettangolare irregolare (m. 1,35 $\times 0,60 \times 1,20$ in lunghezza, altezza e profondità). A m. 3,60 dal suo imbocco, si apre sulla destra, la cella A (larghezza alla base m. 1,40/1,60 al centro $\times 2,80$ altezza $\times 2,10$ profondità); a m. 5, sulla sinistra, la cella $B$ (larghezza alla base $\mathrm{m} .1,70 \times 2$ altezza $\times 1,40$ profondità). Entrambe di forma semiellittica, hanno la copertura ad ogiva tronca $\mathrm{e}$ sono sopraelevate rispetto al piano di calpestio della camera (la A di $\mathrm{m}$. 0,40). In questa, le pareti aggettano fortemente verso l'alto con l'impiego di blocchi variamente poliedrici, di grandi e medie dimensioni, ai quali si interpongono, particolarmente nel lato destro, lastre appiattite e irregolari. Il legame avviene ad incastro con abbondante pietrame minuto. Si contano tredici/quindici filari. La copertura è ad ogiva tronca con un'altezza crescente da m. 2,10 dopo l'architrave d'ingresso fino a m. 5/6 al centro della camera dove chiude a cuneo. Nel limite di fondo si presenta di nuovo l'ogiva. A m. 1,40 dalla cella B, si ha l'innesto della scala $(m .1,20)$ che con una rampa di gradini, si sviluppa sulla destra parallelamente al contorno esterno, con un andamento circolare. La copertura è a lastroni piatti, ascendente verso l'alto. Conduce al corpo a tholos costituito da una camera circolare (diametro m. 3,20). Quanto rimane di tutta la costruzione ci consente di definirla in buon stato di conservazione se si pensa che la struttura del protonuraghe si conserva intatta nei particolari ma nel suo complesso essa è pericolante con probabile situazione di crollo. E'visibile la differenza della tecnica esecutiva del protonuraghe rispetto a quella del corpo a tholos. La prima è un'opera rozza, con l'uso di blocchi di basalto, disposti senza un preciso ordine filarico con abbondante rinzeppatura che contrasta con quella più curata della struttura aggiunta dove forte e pronunciata è l'inclinazione delle pareti, costituite da filari di blocchi più regolari sia per forma che per dimensioni. Più armonico è anche il profilo curvilineo che cura maggiormente l'adesione degli incastri tra i blocchi 


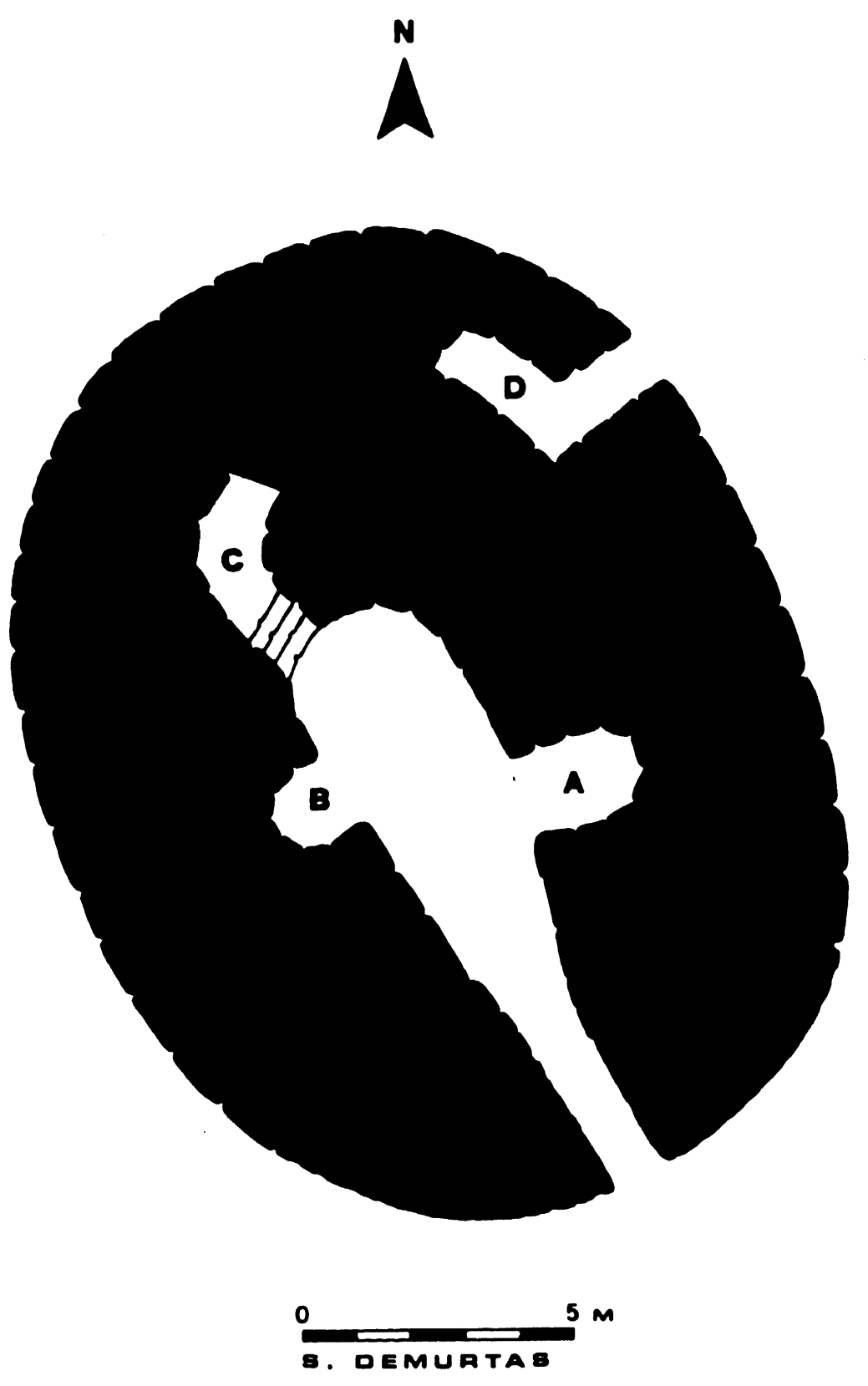

TAV. 4.-Naviforme: Orgono Ghilarza (OR) Pianta (Ril. e Dis. S. Demurtas).

T. P., 1991, n² 48

(c) Consejo Superior de Investigaciones Científicas 
di base e quelli superiori dove non è necessario l'uso di zeppe di rincalzo. In prossimità dell'ingresso, si calcola l'altezza di m. 6,50 con otto filari; al di sopra si adagia il secondo corpo costruttivo per un'altezza di m. 3,70 calcolabile nel lato volto a Nord, con otto/nove filari residui. Della camera a tholos manca completamente la copertura e la scala d'accesso alla terrazza. Attorno al corpo primitivo si conserva un rifascio dove si apre, nel lato a Nord-Nord-Est, una cella a svolta angolare. In tale rifascio si nota una differenza di tecnica muraria. I massi sono talvolta sbozzati anche se in filari irregolari e discontinui.

BIBLIOGRAFIA: (Centurione, 1866: 191; E.E.M., 1922: 116; Contu, 1981: 23, figs. 10-11; MancaDemurtas, 1984 a: 635, 637 e 1984 b: 174; Manca, 1985: 16-17, figs. 2-3).

\section{Crastu A-Soddl-OR (Tav. I, Tav. V, 1, 2)}

E'situato a m. 253 d'altitudine, su un altopiano. A m. 350 ad Est, interposto nel vallone sottostante, scorre il rio Messe Cappai. (F. 206, quadr. II, N-E, Ghilarza).

Il nuraghe presenta due ingressi, camera "naviforme», quattro ambienti contrapposti in coppia (A, B, C, D), vano di disimpegno (E) e vano scala. Ha un contorno quasi circolare con diametro maggiore di $\mathrm{m} .15$ e minore di m. 14,50. La parete in cui si aprono gli ingressi, volta ad Est-Sud-Est, è leggermente tronca. L'ingresso di Est-SudEst (larghezza alla base m. 1,20 × 1,70 altezza) dà adito alla camera principale, di forma "naviforme" mentre l'ingresso di Sud-Est (larghezza alla base $\mathrm{m}$. $1,10 \times 2$ altezza) immette all'ambiente $\mathrm{E}$, di profilo tronco-ellittico. Hanno sezione trapezoidale e sono sormontati ciascuno da un lastrone di architrave $(\mathrm{m} .1,10 \times 0,40 \times 0,80 ; \mathrm{m} .1,50 \times 1,30 \times 0,80$ in lunghezza, altezza e profondità). La camera, larga al suo imbocco m. 1,20, si allarga gradatamente per un percorso di m. 4,60 dando spazio all'apertura di due ambienti (A e D) che si fronteggiano. Tutta la camera è lunga m. 11,50. Ad una distanza di m. 2,30 da A (larghezza alla base $\mathrm{m}$. 1,25 $\times$ 1,75 altezza $\times 2,55$ profondità) si apre la cella $B$ (larghezza alla base $\mathrm{m}$. $0,85 / 0,35$ in alto $\times 1,50$ altezza all'imbocco/2,05 al centro $\times 2,60$ profondità); a sinistra, di fronte a $\mathrm{B}$, la cella $\mathrm{C}$ (larghezza alla base $\mathrm{m} .1,10 / 0,80$ in alto $\times 1,50$ altezza all'imbocco/2,20 al centro $\times 3,10$ profondità che dista $\mathrm{m}$. 3 dall'ambiente $\mathrm{D}$ (larghezza alla base $\mathrm{m}$. $0,90 / 0,45$ in alto $\times 1,50 / 2,20$ altezza $\times 3,40$ profondità). Esse hanno una forma ad ellisse tronca alla base con blocchi orizzontali di varie dimensioni piuttosto irregolari che contornano $\mathrm{i}$ lati. Sono sormontate da un lastrone di architave (A: $\mathrm{m} .1,10 \times$ $0,30 \times 0,70$; B: $\mathrm{m} .1 \times 0,40 \times 0,90$; C: $\mathrm{m} .1,30 \times 0,15 \times 0,80 ; \mathrm{D}: \mathrm{m} .1,05 \times 0,30 \times 0,60$ in lunghezza, altezza e profonditá). Nella cella C l'architrave dell'ingresso è costituito da una lastra molto piatta con uno spessore di $\mathrm{m}$. 0,15 ed è sormontato da un finestrino di scarico $(\mathrm{m} .0,45 \times 0,30)$. Tutte le celle risultano sopraelevate di circa $\mathrm{cm}$. 30 rispetto al piano di calpestio della camera, con un pavimento lastricato. In esse la copertura è a piattabanda. A circa m. 4 dal l'ingresso del vano principale, quasi ad un terzo della parete, si apre a Sud-Est il vano E, con ingresso sopraelevato. E'un vano di disimpego per l'innesto della scala che si apre lateralmente sulla destra, a m. 1,30 dalla soglia. La scala (m. $0,80 / 1)$ ha un andamento sinuoso e segue il contorno esterno. Sulla sua destra, a m. 2,30 dal tratto iniziale, si apre nella massa muraria un piccolo spazio rettangolare che dà luce dall'esterno. La copertura è a piattabanda gradonata fino al pianerottolo mentre nella parte più alta che porta al piano superiore è a piattabanda regolare con lastroni orizzontali. All'interno della camera naviforme le pareti aggettano fortemente verso l'alto. Si ha una disposizione obliqua dei blocchi in una linea ascendente verso il centro della camera stessa dove si raggiunge l'altezza massima di $\mathrm{m}$. 5 contro i m. 1,70 dell'ingresso. La copertura in parte è a piattabanda regolare e a gradino rovescio, in parte ad ogiva. Il monumento si conserva in discrete condizioni per un'altezza massima di m.6 nell'arco da Nord-Est a Sud dove si contano residui dieci/undici filari. Della parete volta ad Ovest, la più rovinata, è visibile il riempimento con pietrame minuto. L'opera, nel suo complesso,risulta abbastanza curata. La tecnica muraria è a filari con l'impiego di grossi massi di basalto variamente rinzeppati negli interstizi. 

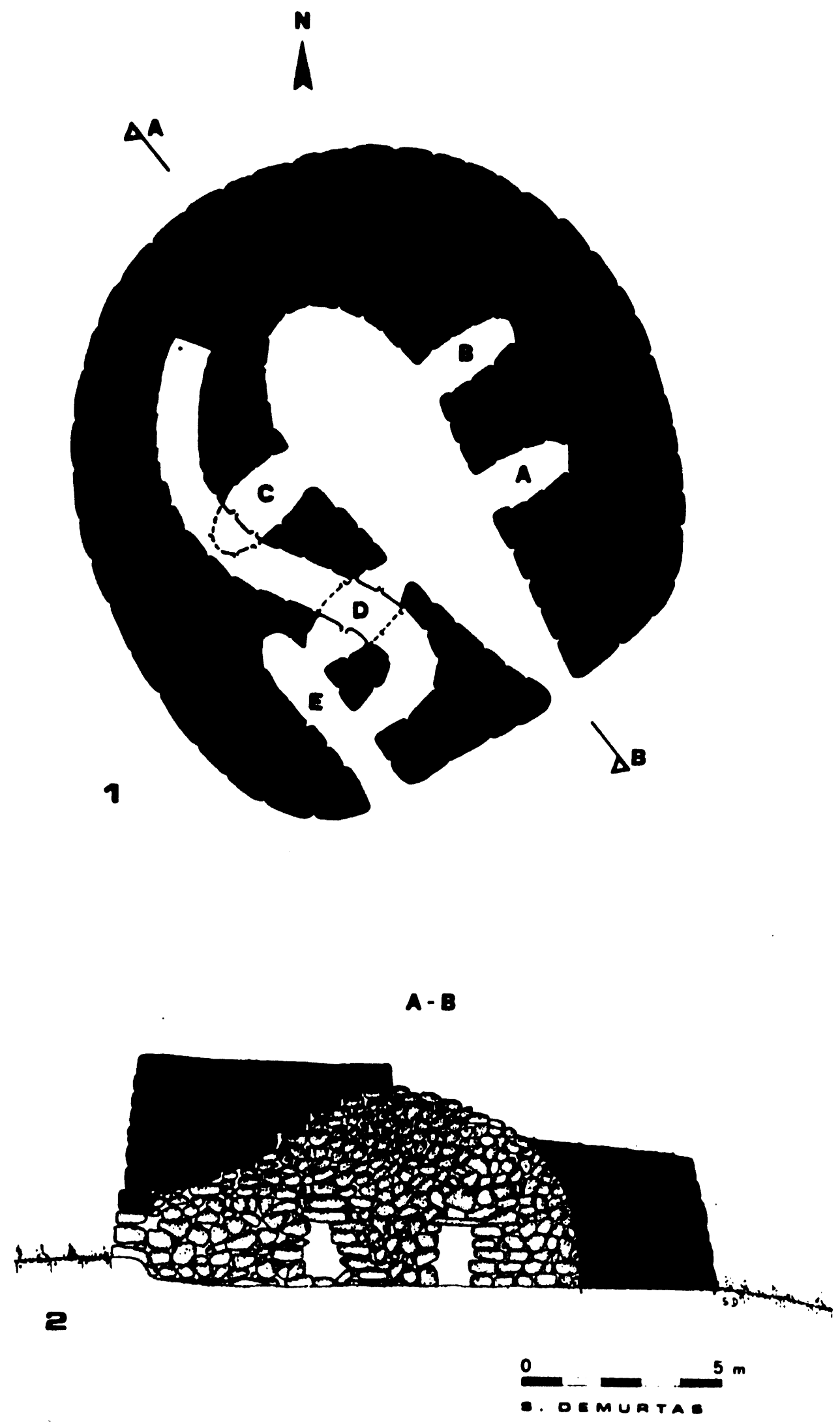

Tav. 5.-Naviforme: Crastu A Soddi (OR). 1) Pianta. 2) Sezione. (Ril. e Dis. S. Demurtas).

T. P., 1991, $\mathrm{n}^{2} 48$

(c) Consejo Superior de Investigaciones Científicas

http://tp.revistas.csic.es Licencia Creative Commons 3.0 España (by-nc) 
BIBLIOGRAFIA: (Centurione, 1886: 188-190, figs. XXIII, XXIV, XXV: E.E.M., 1922: 116; Maxia, 1970: 400; Santoni, 1980: 160, fig. 152; Lilliu, 1982: 15, fig. 4, 2; Manca-Demurtas, 1984 a: 635, 636, fig. 16 e 1984 b: 174, 179, fig. 8, d; lidem, 1987: 406).

\section{ANALISI STRUTTURALE}

La ricerca della figura planimetrica di base viene ad essere strettamente legata ad esigenze statiche e modalità costruttive che presumibilmente porteranno ad una semplificazione di linee. Dall'andamento del tutto irregolare con profilo esterno concavo-convesso (Sa Jacca) (che verrebbe a nascere da un tentativo di utilizzare supporti di roccia naturale esistenti, evidenziando la preocupazione costruttiva) si dovrebbe passare a quello elittico irregolare (Corcove); trapezoidale irregolare (Fodde); tronco-ovoidale con direttrice trasversale (Conculas); tronco-ellittico con attraversamento longitudinale (S. Antine e Campu); ellittico quasi regolare (Orgono); e quasi circolare (Crastu A).

All'interno, la forma della chiglia rovesciata o a carena, è ipotizzabile come un'idea che, in questi nuraghi, si determina con un cambiamento strutturale del corridoio, elemento di passaggio. Probabilmente per i costruttori la trasformazione in camera è il risultato di esperienze precedenti, quindi un punto di arrivo. Il problema per loro non è la pianta interna (e questo lo dimostra la varietá planimetrica: essa è il risultato di un graduale tentativo per lo svuotamento della massa), ma il modo con cui risolvere il problema della copertura. Dovrebbe allora considerarsi basilare la sicurezza dal punto di vista statico e il risparmio costruttivo: pertanto come prima risultanza il corridoio non attraversa la massa muraria. Inoltre la sua apertura decentrata ha un'importanza estremamente significativa. Nel Conculas, tagliando l'asse trasverso della costruzione, viene a permettere l'espansione degli ambienti nell'asse longitudinale con un sistema ortogonale. Nel Corcove, tagliando eccezionalmente l'asse longitudinale, determina un'asimetria di volume rispetto all'intera massa, ma con tale spotamento si ottiene una maggióre porzione di spazio di quella che si sarebbe ottenuta intersecando l'asse trasverso. Così si trova avvantaggiata l'espansione a raggera delle celle A, B, C. Esse si aprono nella camera principale. Le celle rappresentano degli spazi sussidiari in un ambiente principale la cui particolarità è quella di avere un corto corridoio e una camera allungata e svasata ad ellisse. Il suo progressivo allargamento determina una differenziazione tra corridoio e camera e la disposizione degli ambienti nella parte di maggiore ampiezza, implicando presumibilmente la sostituzione della copertura a lastroni piani con quella ad ogiva tronca. I brevi corridoi del Sa Jacca, Corcove, Fodde, S. Antine e Campu potrebbero indicare il perdurare ancora dell'elemento-corridoio come piccolo vano di disimpegno reso talvolta, come nel Sa Jacca, più funzionale perché arricchito di un'altra apertura (b) che potrebbe ritenersi o un ingresso o una finestra per luce.

Al momento non si conoscono nei "naviformi" esemplari con un doppio ingresso contrapposto, usuale nei protonuraghi della I Fase o di tipo A (8). Un caso differenziato è il Crastu A con due ingressi sulla stessa fronte, giustificabili nell'ambito della propria funzione: uno di accesso alla camera principale, l'altro al vano di disimpegno per il piano superiore. La parete di fondo, perpendicolare all'asse del corridoio, presenta diversità: nel nuraghe Sa Jacca essa è risparmiata nella roccia (9), nel Fodde, nel Conculas, nel S. Antine e Campu, nel Crastu A chiude il margine murario della camera stessa in forma absidale, nel Corcove è ricavata la cella $\mathrm{C}$, in Orgono si apre la scala. I costruttori scoprono un'utilizzazione differente nello sfruttamento dello spazio mentre l'innalzamento e la spinta verso l'alto dei volumi vuoti li porta a impratichisi sulla tecnica di chiusura verso l'ogiva tronca badando a bilanciare le spinte e le forze.

Sembrerebbe un momento importantissimo quando nel Sa Jacca aprono il finestrino di scarico

(8) Manca-Demurtas 1984 a: 634-635, figs. 9, 10, 11, 12, 13 e 1984 b: 174-175: 176, fig. 6, a, b, c, d: 177 , fig. 7 a, b, c, d: 179 fig. 8 , a.

(9) Un confronto di tale perizia si può fare con la parete del corridoio, a sinistra di chi entra, del monumento di Torre in Corsica (Grosjean, 1959: 15-40). 
al di sopra dell'architrave: sistema che adotteranno anche dopo nel Crastu A, nell'architrave della cella $\mathrm{C}$ che ha uno spessore minimo di $\mathrm{cm}$. 15, per sostenere meglio il peso della massa portante in concomitanza con la scala (10). Tale criterio di presunta evoluzione avrebbe se non altro il sostegno di uno schema accettabile dal punto di vista logico, concettuale e funzionale, altrimenti non percettibile in questa sequenza.

Dall'analisi del Crastu A parrebbe ricavarsi la cognizione che esso sia la massima espressione che suggerisce nella sua modalità costruttiva il progredire dell'idea della tholos. Pertanto costituirebbe l'esemplificazione del passaggio dal corridoio fino al determinarsi di uno spazio più ampio.

Potremmo affermare che mentre nel protonuraghe della I Fase abbiamo riscontrato una semplice abilità costruttiva (anche se con momenti di incertezza) in questi della II Fase si evidenziano espressioni architettoniche più evolute che si esaltano con la forma interna della camera allungata e soprattutto per l'armonia delle linee. Il vuoto interno si equilibra in un'armoniosa intersezione di due curve innalzantesi con un andamento ampio nel senso longitudinale e ad ogiva tronca nel senso trasversale (11).

Pertanto si potrebbe concludere che le maestranze hanno acquisito coscienza di un vuoto interno utilizzabile, con conseguente capacità di svuotamento e precisione nella individuazione di punti di maggior carico, impostazione del tema fondamentale dell'equilibrio delle masse. La sovrapposizione in verticale nel nuraghe Orgono dei due filoni costruttivi sembrerebbe inquadrarsi in tale schema di lettura.

\section{S. DEMURTAS}

\section{PROBLEMATICA}

Il problema principale è verificare se tale tipologia costituisca un'evoluzione del protonuraghe verso la forma con la tholos o se si tratta del protonuraghe che si modifica a seguito dell'introduzione del contatto di un apporto esterno come taluno (12) riterrebbe plausibile. In una civiltà come quella nuragica, caratterizzata da una intensa dinamica sociale (13) e da mutamenti di modelli architettonici (14) pare particolarmente limitativo parlare di dati ad un solo livello, costituito fondamentalmente da fonti letterarie, per altro indirette, o ritenere un quadro completo e definito quello risultante da parzialitá di conoscenze e conseguente povertà di elaborazione (15). Chi cerca un'impossibile unità in un elemento esterno che "viene accolto in funzione della sua rappresentatività e del suo prestigiow (16) rischia di appellarsi ad una situazione troppo rigida. Considerando il modello del nuraghe a corridoio "fluttuante e disorganico nell'elaborazione degli schemi" "rispetto a quello del nuraghe a tholos regolarizzato a tutti gli effetti» (17) vede in esso un fenomeno avulso da un contesto altrimenti identificabile, e, pertanto, isolato nel suo processo evolutivo cosi da essere visto come «frattura" (18).

La presenza e la distribuzione geografica di un numero sostanzialmente rilevante di protonu-

(10) Manca-Demurtas, 1984: 636 e 1984 b: 180.

(11) Siamo grati all'Ing. Carlo Caboni per le lunghe e fruttuose discussioni a cui non poco deve l'aver elaborato questa linea interpretativa.

(12) Bernardini, 1985: 45-46, nota 8.

(13) Lilliu, 1986: 13.

(14) Lilliu, 1982: 30.

(15) La problematica sul fenomeno è condotta con maggiore ampiezza e rigore rispetto ad analisi semplicistiche e preconcette come mostrano i più recenti studi (Manca-Demurtas, $1984 \mathrm{a}, 1984 \mathrm{~b}, 1987$ ). Ci si augurerebbe minor ricerca di novità a tutti i costi e un più modesto e più funzionale impegno nella documentazione e migliore conoscenza di specifici campi di indagine.

(16) Bernardini, 1985: 52.

(17) Bernardini, 1985: 45.

(18) Bernardini, 1985: 45, cfr. supra nota 15.

\section{T. P., $1991, \mathrm{n}^{2} 48$}


raghi (19) rispetto a quello conosciuto fino ad alcuni anni fa (20), ci fa pensare all'esistenza di un fenomeno costruttivo nel suo kincipit» e alla sua diffusione in un territorio sempre più vasto che va ad interessare tutte le zone della Sardegna.

Carattere peculiare dominante è questo del protonuraghe, conseguente evoluzione delle esperienze precedenti delle genti neolitiche. Queste, furono presumibilmente spinte dalla necesità e dall'esigenza di creare una struttura elevantesi su alture per essere viste, non essendo più sufficenti i rilievi naturali, come presumibile punto distintivo di un possibile ambito territoriale fra le varie tribù. La ricerca, quindi, dell'elevazione del punto più alto, le condurrà ad acquisire conoscenze più dirette, in primo luogo, del territorio e in un momento che può essere pensato anche concomitante, di nozioni molto concrete e pratiche legate all'architettura. La necessità, quindi, del "tumulo protetto" (21) fa sorgere l'idea di un paramento di pietre a filari avente soltanto la funzione di tenuta e di contenimento. La tecnica con cui viene realizzato questo paramento, per quanto primitivo, ha certamente dato l'ispirazione ai costruttori di poter realizzare qualcosa con una capacità statica autonoma. Probabilmente le stesse capanne hanno potuto suggerire l'idea di come poteva essere costruito questo muro, con l'evoluzione della tecniche costruttive (22) anche se antecedentemente, nel Calcolitico, compaiono sistemi fortificati (villaggio con muraglia) in Sardegna e in ambito Mediterraneo (23).

La complessità data dalle soluzioni architettoniche sia in elevato che in pianta dei nuraghi fa pensare a delle maestranze preparate secondo una sequenza plausibilmente predeterminata. Pertanto essa si configurerebbe secondo varie direttrici: 1) scelta del luogo, 2) tecnica di costruzione, 3) creazione del vuoto. Le fasi verrebbero cosi evidenziate: dal semplice paramento esterno, pieno, di Monte d'Accoddi (24) si passa al tipo semplice di protonuraghe dove, probabilmente non esiste ancora la scala. O si usano scale esterne mobili o è lo stesso corridoio che a mò di rampa ascendente, assume tale funzione. E'visibile questo in alcuni nuraghi (25). Poi, nel protonuraghe, caratterizzato da un paramento esterno, di forme varie, dal riempimento del tumulo con pietre e terra e dal corridoio diametrale di base (I Fase, tipo A) (26), è ipotizzabile il verificarsi di un mutamento interno con la necessità di avere spazi più larghi (27). L'ampiezza dei vani sempre più complessi (28) porta alla conoscenza del valore statico del paramento inclinato: oltre che sostenere

(19) Rispetto alla situazione rilevata in Manca-Demurtas, 1984a: 632 (180), si è arrivati nel divenire attuale (e perciò anche implicitamente parziale) a oltre 250 individuabili.

(20) Santoni, 1980, fig. 133. (70).

(21) Il termine indica una specializzazione funzionale già incipiente nello schema felicemente colto in contesto funerario dall'Arnal, 1956: 530.

(22) Elemento già lucidamente sottolineato, con rigore, nella struttura arcaica di Sa Corona dall'Atzeni, $1966: 121$.

(23) Monte Baranta. Olmedo, Sassari, Contu, 1962: 640-641; Moravetti, 1979: 334; Contu, 1981: 64; Moravetti, 1981: 281-290; Idem, 1984: 60; Atzeni, 1988: 452; Moravetti, 1988: 528-529.

Monte Ossoni. Castelsardo, Sassari, Moravetti, 1979: 332-333; Idem, 1981: 287; Idem, 1984: 60; Atzeni, 1988: 452; Moravetti, 1988: 528-529.

Sa Punta 'e S'Arroccu. Chiaramonti, Sassari, Tanda, 1984: 202, nota 420; Moravetti, 1984: 60; Idem, 1988: 528.

Monte Mazzolu. Arzachena, Sassari, Maetzke, 1962: 651-663; Puglisi-Castạldi, 1966: 90-93, fig. 16.

Lasasai. Orani, Nuoro, Manunza, 1984: 400-401.

Crastu Orgiu. Esterzili, Nuoro, Contu, 1981: 64; Idem, 1988: 448.

Fenomeno di cui emergono attualmente esiti peculiari per comprendere le motivazioni della fase di transizione che elabora il modello del protonuraghe quale struttura portante fino alla concezione dell'effetto «anello" nella tholos, attraverso il "naviforme», per raggiungere l'equilibrio totale del monumento.

Da recenti e dirette acquisizioni si segnala il complesso fortificato di Crastu (Soddi, Oristano) (inedito) mentre per l'indicazione della muraglia in località Pedra Oddetta (Macomer, Nuoro) (inédito) ringraziamo Caterina Bittichesu, Isabella Paschina e Santino Lai ai quali pure si deve la segnalazione di alcuni protonuraghi nel territorio di Macomer.

(24) Contu, 1984: 600.

(25) Gazza e S. Caterina (Bolotana-NU); Corongiu Marxi (Gergei-NU); Brunku Madugui (Gesturi-CA); Peppe Gallu (Uri-SS): Manca-Demurtas 1984 b: 178.

(26) Manca-Demurtas, 1984 a: 635 e 1984 b: 174.

(27) Fruscos (Paulilatino-OR), Funtana Suei (Norbello-OR): Manca-Demurtas, 1984 a, figs. 9, 10 e 1984 b: 176, fig. 6, a; Ono (Dualchi-NU), Manca-Demurtas, 1984 b: 177, fig. 7 a.

(28) Canchedda (Ghilarza-OR), Ulinu (Sedilo-OR): Manca-Demurtas, 1984 a, figs. 12, 13 e 1984 b: 176, fig. 6, c: 177, fig. 7, b; Mene (Macomer-NU), S'Ulivera (Dualchi-NU), Cassaros (Torralba-SS): Manca-Demurtas 1984 b: 177, fig. 7, c, d: 179, fig. 8, a. 
il peso proprio resiste alla spinta, ma nel rapporto massa-spazio si ha la consapevolezza che la superficie di solidità è sproporzionata rispetto a quella utile (29), pertanto lo svuotamento si farà sempre più marcato. Si configurerebbe un'evoluzione graduale nel tempo che, attraverso tentativi, condurrà le maestranze a normare particolarità costruttive.

Se ci si sposta da un piano meramente teoretico alla ricerca di documenti archeologici in senso lato, si nota che, sulla scorta del materiale rinvenuto nella parte alta (30) del protonuraghe Fruscos, verremmo a trovarci con una datazione molto alta, al $1904 \pm 104$ (31).

Saremmo di fronte, probabilmente, ai primi tentativi di costruzione ciclopica a filari con l'impiego di blocchi accostati in maniera spesso caotica, ma sempre sovrapposti in una successione di sbalzi aggettanti verso l'interno della massa muraria, in modo che l'intero paramento esterno presenti una caratteristica inclinazione. Rilevante appare, tuftavia, sin dal successivo momento della prima fase, l'applicazione del principio della sovrapposizione degli aggetti per costruire il paramento interno, creando il vuoto dei corridoi. E'questo il momento più significativo, dal punto di vista costruttivo. Si tratta della percezione, prima, e la consapevolezza, poi, di poter creare il vuoto dei vani o delle celle. E'l'evoluzione del paramento interno a svilupparsi, presentando una continuità di applicazioni.

Avremmo cosi una I Fase costruttiva con una seriazione tipologica (Tipo A) ed una II Fase (Tipo B) (32). Nel tipo A la larghezza del corridoio, generalmente, è costante per tutta la lunghezza (33), comincia a variare nel tipo $B$, con un allargamento delle pareti laterali, con direttrice prevalente, quella longitudinale. Pertanto l'evoluzione si configurerebbe attraverso i seguenti passaggi, da considerarsi latamente postulabili: Sa Jacca, Corcove, Fodde, Conculas, S. Antine e Campu, Orgono, Crastu A. La sovrapposizione in senso verticale della camera a tholos nel nuraghe Orgono sarebbe da ritenersi un momento secondario, in una fase di riutilizzo del monumento. La presenza in questo nuraghe del rifascio potrebbe giustificarne il suo uso che è quello di camminamento, essendo evidente (proprio nel tratto del crollo) come lo stesso sia stato costruito per ampliare la base e consentire, quindi, la costruzione della camera superiore (34).

Servendoci dei dati strutturali e architettonici dei protonuraghi in esame si evidenzia una tradizione che parrebbe in grado di elaborare strutture che tendono ad ampliare spazi interni, all'interno della massa compatta, con una tecnica di copertura sempre più simile a quella della tholos. Quindi, potremmo affermare che, nelle grandi linee, questa tipologia di protonuraghi con camera "naviforme" definisce un momento caratterizzante nella formazione della tholos, ine per i costruttori si configura come un'esigenza statica. In effetti elementi che accomunano sono: la copertura ogivale (che in questi casi è ancora in fase di perfezionamento), l'unico ingresso e l'ampiezza dello spazio interno.

I costruttori, sperimentando la capacità delle pietre a chiudere ad ogiva tronca, mettono in rapporto di giusto equilibrio statico l'altezza del monumento con la larghezza, in fun:ione, poi anche di carichi sovrastanti. Ma ben presto si accorgeranno che il principio della copertura a gradino rovescio che, qui, si sviluppa in senso longitudinale, presenta nei punti di minor curvatura, un'evidente debolezza statica rispetto alla parte trasversale in quanto, nella parte allungata, il paramento assolve alla sua funzione solo per effetto della gravità, mentre nei blocchi della parte a curvatura decisa, la resistenza viene garantita anche per effetto dell'aderenza. Pertanto con i monumenti definibili "naviformi" non si ha un completo equilibrio di spinte, poichè nelle parti a curvature dolci si

(29) Manca-Demurtas, 1984 a: 638 e 1984 b: 180 . E'doveroso ringraziare gli amici Dott. Giovanni Tore per i suoi preziosi consigli e l'Ing. Ermanno Scalas per l'elaborazione dei calcoli matematici.

(30) Manca-Demurtas, 1984 a: 639 e 1984 b: 184.

(31) Manca-Demurtas, 1987: 499, nota 33.

(32) Manca-Demurtas, 1984 a: 634 e 1984 b: 174. La ricerca in divenire ha permesso di individuare i seguenti protonuraghi con camera naviforme: Erighighine B, Otzilo, Sa Mura, Urigu-Aidomaggiore (Oristano): Ziringone Paulilatino (Oristano); Carrarzu Iddia B-Mulargia-Bortigali (Nuoro); Sas de Meana (Sorres Meana)- Macomer (Nuoro); Talei Sorgono (Nuoro).

(33) Manca-Demurtas, 1984 a: 634 e 1984 b: 175.

(34) Al momento non parrebbe un caso isolato. Un confronto si può fare col rifascio dei protonuraghi Pobulus (Genoni-NU) e S'Elighe (Sindia-NU).

\section{T. P., 1991, $\mathrm{n}^{2} 48$}


presentano problemi statici, mentre in quelle a curvature più accentuate si evidenzia una grande capacità portante.

Ancora si può notare nel loro sviluppo un'evoluzione significativa nella posizione del vano allungato, per cui con quelli del I periodo della II Fase lo troviamo in una di tipo planimetrico decentrato, mutuata presumibilmente da quella del corridoio del periodo precedente (I Fase). In questa, la posizione del corridoio è così determinata per favorire la garanzia statica del piano abitativo superiore realizzato da capanne prima con tetto stramineo (35) poi con copertura a tholos (36) oppure del nuraghe a tholos (37).

Si evidenzia così che le costruzioni superiori , evidentemente importanti, inizialmente dovevano insistere in una zona piena del monumento; mentre con l'evolversi delle forme curve sia planimetriche che altimetriche e quindi con l'affermarsi di concezioni statiche evolute, il vano principale e così pure le sovrastanti strutture vengono gradatamente sistemate verso il centro, trovando i costruttori, in un processo di innalzamento, la capacità tecnica di ottenere dei vani inferiori di notevole ampiezza e la simultanea padronanza della solidità strutturale per reggere a forti carichi superiori centrati e quindi, come risultante, la tholos.

Pertanto si potrebbe affermare che il protonuraghe presenta la varietà senza unità, il nuraghe a tholos la varietà nell'unità.

Va sottolineato che questa elaborazione concettuale è pienamente delimitata e determinata dalla presente possibilità di validi riscontri ovviamente da confrontarsi e fondarsi con maggiore sicurezza e documentazione attraverso ulteriori ricerche su campo e da specifiche indagini stratigrafiche. L'intento dello studio è di proporre un'ipotesi di discussione su un piano di presumibili ipotesi di lavoro.

Pertanto, va ripreso quanto, in verità, non molto ampio, vi è di documentazione archeologica (38). Infatti questa linea di evoluzione esservata nell'analisi strutturale, non parrebbe contraddetta dagli elementi di cultura materiale che si associano a questi monumenti (39). Essi rivelano notevoli affinità con materiali di tradizione Bonnanaro associati agli stessi nuraghi a tholos e alle tombe di giganti.

E'probabile, quindi, che l'affermarsi della tholos non debba essere associata a innovazioni sul piano della cultura materiale: ci troviamo di fronte ad aspetti inseribili nell'ambito della stessa tradizione, fin'ora documentata in rapporto ai protonuraghi.

Diremmo, concludendo, che pur non escludendo l'influenza di un movimento culturale nella definizione della tholos sembrerebbe che questo giunga a rafforzare un concetto architettonicamente già acquisito.

Se l'elemento egeo si fosse imposto con il modello della tholos, non sarebbe assurdo poter ritenere che avesse dovuto interessare tutte le strutture sia civili che religiose (40), cosi non avremmo trovato solo il nuraghe a tholos con l'incidenza che tutti conosciamo.

D'altra parte, alcuni studiosi inglesi (41) in un'interessante comparazione di struttura di cinque

(35) Manca-Demurtas, 1984 a: 638 e 1984 b: 173.

(36) Sumboe (Ghilarza-OR): Manca-Demurtas, 1984 a: 638, fig. 17 e 1984 b: 173: 179, fig. 8, b.

(37) Orgono (Ghilarza-OR): Manca-Demurtas, 1984 a: 638; S'Ulivera (Dualchi-NU): Manca-Demurtas, 1984 b: 177, fig. 7, d.

(38) Manca-Demurtas, 1984 a: 640, figs. 20-24 e 1984 b: 185-187, figs. 14-16.

(39) Anche se recuperati non all'interno $e$ in stratigrafia, il medesimo quadro tipologico sembrano suggerire i pochi frammenti ceramici rinvenuti all'esterno del protonuraghe Crastu A. Le forme vascolari si riferiscono prevalentemente a tegami bassi con orli sia piatti sia arrotondati, talvolta ribattuti all'esterno. Su un frammento si conserva l'ansa nastriforme impostata dall'orlo alla base, e sul fondo di un altro tegame, la traccia di un'impressione a cordone spiraliforme. Fra gli altri frammenti, si riconoscono forme riferibili a ciotole carenate, a collo alto e ad orlo piatto, e a vasi globoidi con orlo semplice o a colletto. I tegami presentano impasti grossolani, con numerosi inclusi silicei, di piccola e media dimensione e superfici lisciate all'esterno e, talvolta, lucidate all'interno. Più curati gli impasti e le superfici delle altre forme vascolari. Il colore ha tonalità che vanno dal rossiccio al brunastro.

Ringraziamo il Dott. Salvatore Sebis per la sua collaborazione nell'analisi di queste ceramiche.

(40) Santoni, 1980: 148.

(41) Cavanagh-Laxton, 1985: 426, 427, 428. 
nuraghi a tholos con altrettante costruzioni micenee, dimostrano, su base scientifica matematica, attraverso l'equazione di forma, la totale diversità delle due modalità di copertura.

L. MANCA DEMurTaS

\section{BIBLIOGRAFIA}

Arnal, J. (1956): «Petit lexique du Megalitisme». Bulletin de la Societè Préhistorique Francaise, Parigi, LIII, n 9: 518.531. Le Mans.

Atzen,, E. (1966): "Il "Nuraghe" Sa Corona di Villagrecan. Atti del XIII Congresso di Storia dell'Architettura (Sardegna) Cagliari 6-12 Aprile 1963, vol. I: 119-124. Roma.

- (1988): «Megalitismo e Arte. L'età del Rame in Sardegnaw. Rassegna di Archeologia Congresso Internazionale l'età del Rame in Europa (Viareggio 15-18 Ottobre 1987) vol. 7: 449-456. Firenze.

BernardinI, P. (1985): "Tholoi in Sardegna: alcune considerazioni». Studi Etruschi, vol. LI-MCMLXXXIII (Serie III): 43-54. Firenze.

CavanaGh, W. G. - Laxton, R. R. (1985): «Vaulting in Mycenaean tholos tombs and Sardinian nuraghi». Paper in Italian Archaeology IV, Patterns in Protohistory, Part. iii. British Archeological Reporter International Series 245: 413-433. Oxford.

Centurione, A. M. (1886): «Studii recenti sopra i nuraghi e loro importanza». La Civiltà Cattolica, vol. IX: 180-191. Firenze.

Contu, E. (1962): «Il nuraghe Monte Baranta in località Su Casteddu o Pala Reale (Olmedo, Sassari)». Studi Sardi, vol. XVII (1959-61): 640-641. Sassari.

- (1981): «L'architettura nuragica. Ichnussa. La Sardegna dalle origini all'età classica: 5-175. Milano.

- (1984): «Monte D’Accoddi-Sassari. Problematiche di studio e di ricerca di un singolare monumento preistorico». The Deya Conference of Prehistory (Early Settlement in the Western Mediterranean Islands and their Peripheral areas), British Archeological Reporter International Series 229, vol. ii: 591-610. Oxford.

- (1988): "Problematiche ed inquadramento culturale. L'età del Rame in Sardegna». Rassegna di Archeologia. Congresso Internazionale l'età del Rame in Europa (Viareggio 15-18 Ottobre 1987) vol. 7: 441-448. Firenze.

GrosJeAn, R. (1959): "Torre (Corse). Monument mégalithique du Bronze moyen». Revue Archeologique, I, II: 15 40. Ajaccio.

LıLLIU, G. (1982): «La civiltà nuragica. Studi e monumenti I». Sassari.

- (1987): "La Sardegna tra il II e il I millenniuo a. C.n. La Sardegna nel Mediterraneo tra il secondo e il primo millennio a. C. Atti del 2 Convegno di Studiw. Un millennio di relazioni fra la Sardegna e i paesi del Mediterraneom (Selargius-Cagliari 27-30 Novembre 1986): 13-32. Cagliari.

MAETZKE, G. (1962): «Scavi e scoperte nella provincia di Sassari e Nuoro (1959-61)" Studi Sardi, vol. XVII: 651663. Sassari.

Manca Demurtas, L. - Demurtas, S. (1984 a): «I protonuraghi (Nuovi dati per l'Oristanese)m. The Deya Conference of Prehistory (Early Settlement in the Western Mediterranean Islands and their Peripheral areas), British Archeological Reporter International Series 229, vol. ii: 629-670. Oxford.

- (1984b): "Observaciones sobre los protonuragues de Cerdeña». Trabajos de Prehistoria, vol. 41: 165-204. Madrid.

- (1987): «Di un tipo architettonico mediterraneo (Talaiot Rafal Roig-Mercadal-Minorca)». La Sardegna nel Mediterraneo tra il secondo e il primo millennio a. C. Atti del 2 Convegno di Studi. "Un millenio di relazioni fra la Sardegna e i paesi del Mediterraneon (Selargius-Cagliari 27-30 Novembre 1986): 493-503. Cagliari.

MANCA, G. (1985): «Il nuraghe Orgono di Ghilarza». Sardigna antiga, numero 3, Nuoro 1984-1985: 16-17. Nuoro.

ManUnza, M. R. (1984): «Notiziario Sardegna. Orani, loc. Lasasai (Prov., Nuoro)». Rivista di Scienze Preistoriche, vol. XXXIX, 1-2: 400-401. Firenze.

Moravetti, A. (1979): «Notiziario Sardegna. Monte Baranta (Olmedo, Prov. di Sassari); Monte Ossoni (Castelsardo, Prov. di Sassari)m. Rivista di Scienze Preistorische, vol. XXXIV, 1-2: 334, 332-333. Firenze.

- (1981): «Nota agli scavi nel complesso megalitico di Monte Baranta (Olmedo, Sassari)». Rivista di Scienze Preistoriche, vol. XXXVI, 1-2: 281-290. Firenze.

- (1984): «Statue Menhirs in una tomba di giganti del Marghinew. Nuovo Bullettino Archaeologico Sardo, n. 1: 41-67. Sassari.

- (1988): «La cultura Monte Claro nella Sardegna settentrionalew. Rc ssegna di Archeologia Congresso Internazionale l'età del Rame in Europa (Viareggio 15-18 Ottobre 1987) vos 7: 528-529. Firenze.

MAXIA, C. (1970): «La civiltà nuragica. Le ultime scoperte». Frontiera, anI I III, n 10; 400 Cagliari.

PUGLISI, S. M. - CASTALDL E (1966): «Aspetti dell'accantonamento culturale nella Gallura preistorica e protostorica». Studi Sardi, vol. XIX (1964-1965): 59-96. Sassari.

T. P., 1991, $\mathrm{n}^{\mathbf{2}} 48$ 
RAmiS y Ramis, J. (1818): «Antigüedades Célticas de la Isla de Menorca desde los tiempos más remotos hasta el siglo IV de la era Cristiana». Maó.

Santon, V. (1966): «Saggio di catalogo archeologico sul Foglio 207 della Carta d'Italia, Quadrante IV, Tav. N.E.», Università di Cagliari, anno accademico 1965-1966 (Tesi di Laurea).

- (1976): «Nota preliminare sulla tipologia delle grotticelle funerarie in Sardegna». Archivio Storico Sardo, vol. XXX: 3-49. Cagliari.

- (1980): «Il segno del poterew. Nur la misteriosa civilià dei Sardi: 141-186. Milano.

TANDA, G. (1984): «Arte e religione della Sardegna preistorica nella necropoli di Sos Furrighesos», vol. II, Sassari.

TARAmelli, A. (1904): "Busachi. Ricerche nelle tombe scavate nella roccia dette "Domu de Janas" in località "Sa Pardischedda" e "Campu Maiore». Notizie Scavi, Fasc. 5: 209-219. Roma.

VenY, C. (1982): "Las Navetas». Geografía e Historia de Menorca, Coordinada por J. Mascaro Pasarius: $177-311$. Ciutadela. 